\title{
Can biomarkers predict chemosensitivity in CRS-HIPEC patients?
}

Josephine Hendrikson ${ }^{1}$, Joey WS Tan ${ }^{1} \&$ Chin-Ann J Ong*,1

${ }^{1}$ Department of Sarcoma, Peritoneal \& Rare Tumours (SPRinT), Division of Surgery \& Surgical Oncology, National Cancer Centre Singapore, 11 Hospital Crescent S169610, Singapore

*Author for correspondence: johnny.ong.c.a@singhealth.com.sg

“Despite favorable results reported in numerous studies involving CRS-HIPEC, significant improvements in systemic chemotherapy have warranted further evaluation on the role of HIPEC in CPM management"

First draft submitted: 5 December 2019; Accepted for publication: 9 December 2019; Published online: 30 January 2020

Keywords: biomarker $\bullet$ chemosensitivity $\bullet$ colorectal cancer $\bullet$ CRS HIPEC $\bullet$ peritoneal metastasis

Colorectal peritoneal metastasis (CPM) occurs in approximately $15-20 \%$ of colorectal cancer patients. Five to ten percent of these patients are identified synchronously during the time of primary tumor resection, while 20$50 \%$ of patients are diagnosed with metachronous CPM [1-3]. The prognosis of CPM is poor and is comparably worse than other sites of metastases, with a median survival of 6 months in untreated cases and 12.7 months with systemic chemotherapy $[2,4,5]$. The introduction of cytoreductive surgery (CRS) combined with hyperthermic intraperitoneal chemotherapy (HIPEC), applied to a group of carefully selected patients, has dramatically improved the outcome of this disease resulting in a median survival of 34.3 months [6]. This treatment modality involves extensive surgery to completely remove all visible tumors, followed with an administration of a high concentration of heated chemotherapy to the peritoneal cavity to eradicate residual microscopic disease $[7,8]$.

\section{Latest Clinical Trials on CRS-HIPEC for CPM}

Despite favorable results reported in numerous studies involving CRS-HIPEC, significant improvements in systemic chemotherapy have warranted further evaluation on the role of HIPEC in CPM management. Two recent Phase III randomized-controlled trials have attempted to investigate the effects of HIPEC in two different settings: in patients with isolated CPM and in an adjuvant setting for high-risk CRC patients.

\section{PRODIGE 7}

The PRODIGE 7 trial was designed to determine the therapeutic benefit of HIPEC for patients with CPM. The results were recently presented at the American Society of Clinical Oncology 2018 conference [9]. In this study, 265 patients with colorectal cancer and isolated peritoneal metastases were enrolled and randomized intra-operatively to the control (CRS and perioperative chemotherapy, $\mathrm{n}=132$ ) or experimental (CRS with oxaliplatin-HIPEC and perioperative chemotherapy, $\mathrm{n}=133) \mathrm{arm}$. The trial found no additional overall survival $(O S)$ or disease-free survival (DFS) benefits in the oxaliplatin-HIPEC group, while 60-day morbidity rate was significantly higher.

\section{COLOPEC}

COLOPEC is a randomized multi-centre trial, which aimed to investigate the efficacy of adjuvant HIPEC in preventing the development of PM in patients with high-risk CRC [10]. Patients $(n=202)$ with locally advanced (T4 stage) or perforated colon cancer were successfully recruited and randomly assigned to standard adjuvant systemic chemotherapy (control arm, $\mathrm{n}=102$ ) or adjuvant oxaliplatin-HIPEC, followed by systemic chemotherapy (experimental arm, $\mathrm{n}=100$ ). No significant difference in peritoneal-free survival at 18 months was reported and postoperative complications were found in patients receiving adjuvant HIPEC.

Future Medicine 
Results from the two trials have indicated that HIPEC with oxaliplatin failed to demonstrate survival benefit among patients with CPM, as well as patients with high risk for developing peritoneal metastasis. Instead, it is associated with morbidities that may be limited to the intraperitoneal use of oxaliplatin. These findings highlighted three essential points. First, conventional clinicopathologic prognostic variables such as peritoneal carcinomatosis index, completeness of cytoreductive score, histologic tumor grade, lymph node status and synchronous liver metastases, have provided great tools for patient selection for surgical indication and prognosis, but not for HIPEC indication. Second, providing standardised HIPEC regimen to all patients may not work due to differences in tumor biology. Third, as individual intraperitoneal chemotherapy regimens used in HIPEC also contribute to the morbidities associated with this treatment modality, it is essential to have stringent selection criteria for patients who are likely to receive the most benefit from this treatment. Thus, there is an urgent need to develop predictive biomarkers that consider the biological characteristics of individual tumor to guide the optimal use of HIPEC in the management of CPM. Only then, can we truly assess the survival benefits conferred by HIPEC.

\section{Prognostic \& predictive biomarkers for CRS-HIPEC in CPM}

Three recent studies endeavored to identify prognostic and predictive biomarkers to evaluate the efficacy of HIPEC in CPM.

\section{BLM}

To establish a predictive biomarker for CPM, Kwakman et al. [11] correlated MMC sensitivity of 12 colorectal cancer cell lines with mRNA expression of 37 genes involved in mitomycin C (MMC) activation pathway and MMC-induced damage repair pathways such as FA-BRCA and ATM-ATR. Low expressions of BLM and BRCA2 were significantly associated with MMC sensitivity at the mRNA level $(\mathrm{p}=0.01$ and $\mathrm{p}=0.02$, respectively). Combined expression levels of the two genes showed equivalent correlation with $B L M$ alone $(\mathrm{p}=0.01)$. Internal validation was performed using immunohistochemistry (IHC) of BLM in FFPE samples from 20 CRS-HIPEC patients who received R1 (tumor $<2.5 \mathrm{~mm}$ ) resection outcome. High BLM was identified as a predictive biomarker of resistance to MMC and poorer survival in CPM patients (median OS, 15 months vs indeterminate for high and low BLM expressions respectively; $\mathrm{p}=0.04)$.

\section{Angiogenesis markers - VEGF \& VCAN}

Sluiter et al. [12] investigated the role of angiogenesis molecules such as VCAN and VEGF in prognosticating CPM patients who underwent CRS and MMC-HIPEC. Expression of these proteins was assessed using IHC of whole tissue specimens from 65 patients. High VEGF and low epithelial VCAN were associated with poorer prognosis (high VEGF vs low VEGF mean survival: 29.2 months vs 38.3 months; $\mathrm{p}=0.035$ and high epithelial VCAN vs low epithelial VCAN mean survival: 36.8 months vs 20.1 months; $\mathrm{p}=0.109)$. After adjusting for clinicopathologic factors, VEGF and epithelial VCAN expression were identified as independent prognostic factors $(\mathrm{p}=0.012$ and $\mathrm{p}=0.042$, respectively) and can potentially be used to identify CPM patients who are at risk of treatment failure following CRS-HIPEC. This study was reported according to the REMARK guidelines [13].

In these studies, potential prognosis and predictive biomarkers are derived through interrogation of genes, which possibly contributed to peritoneal dissemination (such as angiogenesis) and drug resistance mechanisms. While these approaches have been successful, prior knowledge of tumor biology is required to identify candidate genes for further study. Therefore, an unbiased approach utilizing the entire genome could be applied to identify novel molecules.

\section{A set of molecular markers - PAXIP1 \& SSBP2}

A recent study by our group successfully generated biomarkers that predict chemosensitivity to MMC-HIPEC in CPM by harnessing publicly available databases [14]. In this study, genes associated with MMC sensitivity were derived by correlating MMC IC 50 of 35 colorectal cancer cell lines and their expression profiles from Genomics of Drug Sensitivity in Cancer database. The list of genes was further refined by comparing transcriptomic data with survival data across The Cancer Genome Atlas cohort, which yielded four putative MMC chemosensitivity biomarkers (PAXIP1, SSBP2, DTYMK and HMGB1). Validation was performed via IHC on tissue microarray of 62 CPM patients who underwent CRS-HIPEC. Lower expressions of PAXIP1 and SSBP2 were correlated with poorer OS $(\mathrm{p}=0.045$ and $\mathrm{p}=0.140$, respectively). Multivariate Cox regression analysis revealed significant associations between the combined two-marker set and poorer OS and DFS (two dysregulated markers vs one or 
no dysregulated markers: median OS, 18.4 vs 42.3 months; $\mathrm{p}=0.016$; median DFS, 12 vs 22 months; $\mathrm{p}=0.018$ ), performing better than standard clinicopathological prognostic factors such as peritoneal carcinomatosis index and completeness of cytoreductive scores. This study was reported in close adherence to the REMARK guidelines.

We believe that this unbiased approach is useful for future biomarker studies as it can be easily applied to find chemosensitivity signatures for other HIPEC drugs and to peritoneal metastasis from other histological subtypes.

Moving forward, large scale multi-centre and multi-national validation studies are integral to verify the prognostic and predictive roles of these candidate biomarkers. These studies are ideally conducted in a well-defined patient cohort, using samples collected from experienced institutes, and in adherence to REMARK guidelines to effectively make the transition to clinical practice. Simultaneously, research using patient-derived in vitro and in vivo models of CPM is vital to provide better understanding of the biological basis of CPM pathophysiology and in determining efficacy and safety of alternative/new therapeutic agents for HIPEC. Another promising avenue is to employ multiomics integrative analysis to uncover predictive biomarkers and targeted therapeutic targets for CPM. Such research could be facilitated by the use of laser capture microdissection to obtain homogenous tumor cell populations for highly specific biomarkers identification [15].

In conclusion, despite the failures from recent clinical trials to demonstrate benefits of HIPEC, we believe that HIPEC can still be beneficial in the treatment of CPM with the aid of robust predictive biomarkers to identify patients who will benefit from a personalized HIPEC regimen. However, current studies in this area are still at the infancy stage and require a concerted effort among peritoneal surface malignancy groups to systematically identify and validate molecular markers, which are predictive for the diverse chemotherapy agents used in HIPEC.

\section{Financial \& competing interests disclosure}

CAJ Ong is supported by National Medical Research Council Transition Award (NMRC/TA/0061/2017). The authors have no other relevant affiliations or financial involvement with any organization or entity with a financial interest in or financial conflict with the subject matter or materials discussed in the manuscript apart from those disclosed.

No writing assistance was utilized in the production of this manuscript.

\section{Open access}

This work is licensed under the Attribution-NonCommercial-NoDerivatives 4.0 Unported License. To view a copy of this license, visit http://creativecommons.org/licenses/by-nc-nd/4.0/

\section{References}

Papers of special note have been highlighted as: $\bullet$ of interest $\bullet \bullet$ of considerable interest

1. Chu DZ, Lang NP, Thompson C, Osteen PK, Westbrook KC. Peritoneal carcinomatosis in nongynecologic malignancy. A prospective study of prognostic factors. Cancer 63(2), 364-367 (1989).

2. Sadeghi B, Arvieux C, Glehen O et al. Peritoneal carcinomatosis from non-gynecologic malignancies: results of the EVOCAPE 1 multicentric prospective study. Cancer 88(2), 358-363 (2000).

3. Jayne DG, Fook S, Loi C, Seow-Choen F. Peritoneal carcinomatosis from colorectal cancer. Br. J. Surg. 89(12), 1545-1550 (2002).

4. Franko J, Shi Q, Meyers JP et al. Prognosis of patients with peritoneal metastatic colorectal cancer given systemic therapy: an analysis of individual patient data from prospective randomised trials from the Analysis and Research in Cancers of the Digestive System (ARCAD) database. Lancet Oncol. 17(12), 1709-1719 (2016).

5. Verwaal VJ, van Ruth S, de Bree E et al. Randomized trial of cytoreduction and hyperthermic intraperitoneal chemotherapy versus systemic chemotherapy and palliative surgery in patients with peritoneal carcinomatosis of colorectal cancer. J. Clin. Oncol. 21(20), 3737-3743 (2003).

6. Huang C-Q, Min Y, Wang S-Y et al. Cytoreductive surgery plus hyperthermic intraperitoneal chemotherapy improves survival for peritoneal carcinomatosis from colorectal cancer: a systematic review and meta-analysis of current evidence. Oncotarget 8(33), 55657-55683 (2017).

7. Spratt JS, Adcock RA, Muskovin M, Sherrill W, McKeown J. Clinical delivery system for intraperitoneal hyperthermic chemotherapy. Cancer Res. 40(2), 256 (1980).

8. Sugarbaker PH. Peritonectomy procedures. Ann. Surg. 221(1), 29-42 (1995).

9. Quenet F, Elias D, Roca L et al. A UNICANCER Phase III trial of hyperthermic intra-peritoneal chemotherapy (HIPEC) for colorectal peritoneal carcinomatosis (PC): PRODIGE 7. J. Clin. Oncol. 36(Suppl. 18), LBA3503-LBA3503 (2018).

10. Klaver CEL, Wisselink DD, Punt CJA et al. Adjuvant hyperthermic intraperitoneal chemotherapy in patients with locally advanced colon cancer (COLOPEC): a multicentre, open-label, randomised trial. Lancet Gastroenterol. Hepatol. 4(10), 761-770 (2019). 
11. Kwakman R, de Cuba EMV, de Winter JP et al. Tailoring heated intraperitoneal mitomycin C for peritoneal metastases originating from colorectal carcinoma: a translational approach to improve survival. Br. J. Cancer 112(5), 851-856 (2015).

-. First paper to report predictive biomarker for mitomycin C-hyperthermic intraperitoneal chemotherapy (MMC-HIPEC) in colorectal peritoneal metastasis (CPM).

12. Sluiter NR, de Cuba EMV, Kwakman R et al. Versican and vascular endothelial growth factor expression levels in peritoneal metastases from colorectal cancer are associated with survival after cytoreductive surgery and hyperthermic intraperitoneal chemotherapy. Clin. Exp. Metastasis 33(4), 297-307 (2016).

-• Biologically relevant biomarkers that were validated for prognostication in CPM.

13. McShane LM, Altman DG, Sauerbrei W et al. REporting recommendations for tumour MARKer prognostic studies (REMARK). Br. J. Cancer 93(4), 387-391 (2005).

- $\quad$ Provides guidelines for reporting biomarker studies.

14. Shannon NB, Tan JW-S, Tan HL et al. A set of molecular markers predicts chemosensitivity to Mitomycin-C following cytoreductive surgery and hyperthermic intraperitoneal chemotherapy for colorectal peritoneal metastasis. Sci. Rep. 9(1), 10572 (2019).

-. Utilizes an unbiased approach to generate prognostic and predictive biomarkers for MMC-HIPEC in CPM.

15. Ong CAJ, Tan QX, Lim HJ et al. An optimized protocol harnessing laser capture microdissection on matched primary and metastatic colorectal tumours. Sci. Rep. doi:10.1038/s41598-019-55146-2 (2019). 